\title{
RISK BEARING AND THE INSURANCE MARKET
}

\author{
Hans Bühlmann and Hans u. Gerber
}

\section{Introduction}

Stimulated by Karl Borch's paper [3] we have tried to analyze the paper written by K. Arrow [I] in 1953. Contrary to Borch's opinion we have some doubt whether this work contains a theory of insurance as a special case. Nevertheless, it has inspired us to this note, which tries to develop a somewhat more realistic model. As a matter of fact, our development is more in the spirit of another paper by Arrow [2]. We, however, have chosen a more general setup, and we believe that our treatment is also different.

\section{Arrow's Model (Interpreted Freely)}

Arrow considers an economy of exchange with $C$ commodities (labelled $c=\mathrm{I}, \ldots, C$ ) and a "world" that will be in one of $S$ different states $(s=\mathrm{I}, \ldots, S)$. The problem is to distribute the total supply of each commodity $c$ in state $s$ among I individuals in a Pareto-optimal fashion. According to a standard result in economic equilibrium theory every Pareto-optimal allocation can be realized by a system of perfectly competitive markets. The latter means that there are prices $\vec{p}_{s c}$ (the price for a unit of commodity $c$ if state $s$ occurs) and that each individual has a certain amount of money, which he then will spend to maximize his own utility. The beauty of this approach lies in its simplicity: Each individual has his own maximization problem (irrespective of the others). Thus it is enough to focus our attention on a particular individual. Let $y$ denote his spendable money, let $x_{s c} \geq 0$ denote the amount of commodity $c$ contingent to the occurrence of state $s$ purchased, and let $V\left(x_{11}, \ldots, x_{S C}\right)$ denote the "value" (or utility) of this decision.

Then the problem is to

$$
\begin{aligned}
\operatorname{maximize} & V\left(x_{11}, \ldots, x_{S C}\right) \\
\text { subject to } & \sum_{,=1}^{s} \sum_{c=1}^{c} x_{s c} \bar{p}_{s c} \leq y .
\end{aligned}
$$

Arrow's idea is to replace this market by a two stage market. Let $q_{1}>0, \ldots, q_{S}>0$ be arbitrary numbers with $q_{1}+\ldots+$ $+q_{S}=\mathrm{I}$. Here $q_{s}$ is the price of a security ("policy" in insurance 
terminology) of type $s$, which pays one monetary unit if state $s$ occurs and nothing otherwise. Let $p_{s}$ be the price of commodity $c$ when state $s$ has occurred. For consistency set

$$
p_{s c}=\bar{p}_{s c} / q_{s}
$$

The two decisions are now:

a) choice of the securities. Buy $y_{s} \geq 0$ securities of type $s(s=\mathbf{I}$, $\ldots$, S) such that $\sum_{s=1}^{s} y_{s} q_{s} \leq y$.

b) Purchase of commodities after the state s has occurred. Let $x_{s c}$ denote the amount of commodity $c$ that is purchased after the state $s$ has occurred. We must have $\sum_{c=1}^{c} x_{s c} p_{s c} \leq y_{s}+y-\sum_{i=1}^{s} y_{i} q_{i}$. Again, we make our decision in a) and b) to maximize the resulting utility. Obviously, this two stage problem is equivalent to the original problem (I), equivalence meaning that the same commodity bundles can be bought with the same original money amount.

From now on let us assume that the function $V$ is of the form (according to the axioms of vonNeumann-Morgenstern)

$$
V\left(x_{11}, \ldots, x_{S C}\right)=\sum_{s=1}^{s} \pi_{s} V_{s}\left(x_{s 1}, \ldots, x_{s C}\right) \text {. }
$$

Here $\pi_{s}$ is the individual's subjective probability for state $s$, and $V_{s}$ is the utility function that applies when state $s$ occurs. Let

$$
\begin{gathered}
U_{s}(w)=\text { maximum } V_{s}\left(x_{s 1}, \ldots, x_{s C}\right) \\
\text { subject to } x_{s c} \geq 0, \sum_{c=1}^{c} x_{s c} p_{s c} \leq w .
\end{gathered}
$$

Thus $U_{s}(w)$ is the utility of $w$ monetary units in state $s$. With these definitions and assumptions problem a) (optimal choice of the securities) can be isolated as follows:

$$
\begin{aligned}
& \operatorname{maximize} \underset{\sum_{s=1}^{S}}{s} \pi_{s} U_{s}\left(y+y_{s}-\sum_{i=1}^{s} y_{i} q_{i}\right) \\
& \text { subject to } y_{s} \geq 0, \sum_{i=1}^{s} y_{i} q_{i} \leq y \text {. }
\end{aligned}
$$

\section{The Problems of Optimal Coverage}

We shall study in detail the solutions of problems of the type (5). Our assumptions are as follows. a) The $S$ utility functions $U_{s}(y)$ are twice differentiable, such that $U_{s}^{\prime}(y)>0$ and $U_{s}^{\prime \prime}(y)<0$. Thus we assume that the utility functions are risk adverse. b) $q_{1}+\ldots$ 
$+q_{S} \geq$ I. If $p_{s}$ is the probability that the market assigns to state $s$, certainly $q_{s} \geq p_{s}$. Summation over $s$ yields the inequality above. If $q_{1}+\ldots+q_{s}=\mathrm{r}$, (as in Arrows model) we can assume that $\sum_{i=1}^{s} y_{i} q_{i}=y$ without loss of generality in (5). However, in the more interesting case where $q_{1}+\ldots+q_{S}>\mathrm{I}$, this is not true anymore. This suggests that we distinguish the following two problems.

\section{Problem A}

For a fixed $z, 0 \leq z \leq y$, maximize $\sum_{s=1}^{s} \pi_{s} U_{s}\left(y+y_{s}-z\right)$ subject to the constraints that $y_{s} \geq 0$ and $\sum_{s=1}^{s} y_{s} q_{s}=z$.

\section{Problem B}

$$
\begin{aligned}
& \text { Maximize } \sum_{s=1}^{s} \pi_{s} U_{s}\left(y+y_{s}-\sum_{i=1}^{s} y_{i} q_{i}\right) \text { subject to } y_{s} \geq 0 \text {, and } \\
& \sum_{s=1}^{s} y_{s} q_{s} \leq y \text {. }
\end{aligned}
$$

Thus in Problem A the total amount spent for premiums, $z$, is prescribed, while in Problem B it is variable, subject only to the upper bound $y$.

In either case the existence of an optimal solution is clear: The quantity to be maximized is a continuous function of the decision variables $y_{1}, \ldots, y_{S}$, which (in both cases) vary over a compact set.

\section{Solution of Problem A.}

\section{Theorem I}

For any $z(0 \leq z \leq y)$ there is a unique vector $\tilde{y}_{1}, \ldots, \tilde{y}_{S}$ satisfying

(i) $\sum_{s=1}^{s} \tilde{y}_{s} q_{s}=z, \tilde{y}_{s} \geq 0$ for all $s$

(ii) $\frac{\pi_{s}}{q_{s}} U_{s}^{\prime}\left(y+\tilde{y}_{s}-z\right) \leq K$ for all $s$, such that $\tilde{y}_{s}=0$ whenever this inequality is strict.

This vector, and only this vector, solves problem $\mathrm{A}$.

Proof

For $z=0$, the theorem is trivially true. Hence assume $z>0$. To show the necessity of condition (ii), consider a vector $y_{1}, \ldots, y_{S}$ 
for which it is violated. Then there are indices $s, t$ such that $y_{t}>0$, $y_{s} \geq 0$ and

$$
\frac{\pi_{t}}{q_{t}} U_{t}^{\prime}\left(y+y_{t}-z\right)<\frac{\pi_{s}}{q_{s}} U_{s}^{\prime}\left(y+y_{s}-z\right)
$$

Then, by increasing $y_{s}$ and decreasing $y_{t}$ (such that the total premium remains z) the expected utility could be increased. (Note that for this part of the proof we did not need the assumption that the utility functions are risk averse.)

The necessity (and the existence of an optimal solution) show that there is at least one vector $\hat{y}_{1}, \ldots, \tilde{y}_{S}$ that satisfies conditions (i) and (ii) above. Let $y_{1}, \ldots, y_{S}$ be any other vector that satisfies (i). First using concavity from below of the function $U_{s}$, and then (ii), we obtain the following estimate:

$$
\begin{gathered}
U_{s}\left(y+y_{s}-z\right) \leq U_{s}\left(y+\tilde{y}_{s}-z\right)+U_{s}^{\prime}\left(y+\tilde{y}_{s}-z\right) \cdot\left(y_{s}-y_{s}\right) \\
\leq U_{s}\left(y+\tilde{y}_{s}-z\right)+K \frac{q_{s}}{\pi_{s}}\left(y_{s}-\tilde{y}_{s}\right) .
\end{gathered}
$$

Note that the first inequality is strict unless $y_{s}=y_{s}$. By summing (7) over $s$ we see that

$$
\sum_{s=1}^{s} \pi_{s} U_{s}\left(y+y_{s}-z\right) \leq \sum_{s=1}^{s} \pi_{s} U_{s}\left(y+\tilde{y}_{s}-z\right)
$$

with a strict inequality holding unless $y_{s}=\tilde{y}_{s}$ for all $s$. This completes the proof of Theorem I.

\section{Solution of Problem B.}

If $\sum_{i=1}^{s} q_{s}=\mathrm{I}$, solve Problem A with $z=y$. Otherwise, the following result holds.

\section{Theorem 2}

Suppose that $\stackrel{s}{\Sigma}_{s-1} q_{s}>$ r. Then Problem B has a unique solution, which we denote by $\hat{y}_{1}, \ldots, \tilde{y}_{s}$. a) If $\sum_{s=1}^{s} y_{s} q_{s}=y$, it can be characterized by conditions (i) and (ii) in Theorem I with $z=y$. b) If $\sum_{s=1}^{s} \tilde{y}_{s} q_{s}<y$, it is the only vector $\tilde{y}_{1}, \ldots, \tilde{y}_{S}$ that satisfies i) $\tilde{y}_{s} \geq 0$ for all $s$ and

ii) $\frac{\pi_{s}}{q_{s}} U_{s}^{\prime}\left(y+\tilde{y}_{s}-\sum_{i=1}^{s} \tilde{y}_{i} q_{i}\right) \leq \sum_{j=1}^{s} \pi_{j} U_{j}^{\prime}\left(y+y_{j}-\sum_{i=1}^{s} \tilde{y}_{i} q_{i}\right)$ for all $s$, such that $\tilde{y}_{s}=0$ whenever the inequality is strict. 


\section{Proof}

a) If there is an optimal $\tilde{y}_{1}, \ldots, \tilde{y}_{s}$ with $\sum_{s=1}^{s} \tilde{y}_{s} q_{s}<y$, it has to satisfy condition (ii) above. For, if it did not, there would either be an index $s$ such that

$$
\frac{\pi_{s}}{q_{s}} U_{s}^{\prime}\left(y+\tilde{y}_{s}-\sum_{i=1}^{s} y_{i} q_{i}\right)>\sum_{j=1}^{s} \pi_{j} U_{j}^{\prime}\left(y+\tilde{y}_{j}-\sum_{i=1}^{s} \tilde{y}_{i} q_{i}\right),
$$

in which case the expected utility could be increased by increasing $\tilde{y}_{s}$, or there would be an index $s$ such that $\tilde{y}_{s}>0$ and the inequality in (ii) is strict, in which case the expected utility could be increased by a reduction of $\tilde{y}_{s}$. (For the necessity of (ii) we again did not need the assumption that the utility functions are risk averse).

b) Suppose now that $\tilde{y}_{1}, \ldots, \tilde{y}_{S}$ is a vector that satisfies conditions (i) and (ii) of part b) in Theorem 2. Any other decision, say $y_{1}, \ldots, y_{s}$ (where $\Sigma q_{s} y_{s}=y$ is also permissible), can be compared

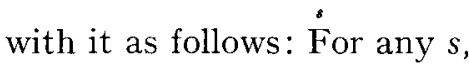

$$
\begin{gathered}
U_{s}\left(y+y_{s}-z\right) \leq U_{s}\left(y+\tilde{y}_{s}-\tilde{z}\right)+U_{s}^{\prime}\left(y+\tilde{y}_{s}-\tilde{z}\right) \cdot\left(y_{s}-\tilde{y}_{s}+\tilde{z}-z\right) \\
\leq U_{s}\left(y+\tilde{y}_{s}-\tilde{z}\right)+\frac{q s}{\pi_{s}}\left(y_{s}-\tilde{y}_{s}\right) \Sigma \pi_{j} U_{j}^{\prime}\left(y+\tilde{y}_{j}-\tilde{z}\right)+ \\
\quad+U_{s}^{\prime}\left(y+\tilde{y}_{s}-\tilde{z}\right) \cdot(\tilde{z}-z), \quad \text { (IO) }
\end{gathered}
$$

with the convenient notation $\tilde{z}=\Sigma \tilde{y}_{i} q_{i}, z=\Sigma y_{i} q_{i}$. Multiplying both sides by $\pi_{s}$, and summing over $s$, we get

$$
\sum_{s=1}^{s} \pi_{s} U_{s}\left(y+y_{s}-z\right) \leq \sum_{s=1}^{s} \pi_{s} U_{s}\left(y+\tilde{y}_{s}-z\right) .
$$

Furthermore, this inequality is strict unless $y_{s}=\tilde{y}_{s}$ for all $s$, which shows the uniqueness of any optimal solution satisfying (ii).

\section{How to Find the Solutions.}

To find the solution of Problem A, first relabel the states such that

$$
\frac{\pi_{1}}{q_{1}} U_{1}^{\prime}(y-z) \geq \frac{\pi_{2}}{q_{2}} U_{2}^{\prime}(y-z) \geq \ldots \geq \frac{\pi_{S}}{q_{S}} U_{S}^{\prime}(y-z) .
$$

Now we choose $y_{1}$ such that

$$
\frac{\pi_{1}}{q_{1}} U_{1}^{\prime}\left(y+y_{1}-z\right)=\frac{\pi_{2}}{q_{2}} U_{2}^{\prime}(y-z)
$$


Then we increase $y_{1}$ and choose $y_{2}$ such that

$$
\frac{\pi_{1}}{q_{1}} U_{1}^{\prime}\left(y+y_{1}-z\right)=\frac{\pi_{2}}{q_{2}} U_{2}^{\prime}\left(y+y_{2}-z\right)=\frac{\pi_{3}}{q_{3}} U_{3}^{\prime}(y-z)
$$

etc. Thus, gradually we increase the coverage, from left to right, until the total premium reaches the level $z$. Clearly, the resulting coverage will satisfy properties (i) and (ii) of Theorem I.

For the further discussion, let $\tilde{y}_{1}, \ldots, \tilde{y}_{S}$ denote the optimal coverage if the premium equals $z$, hence

$$
U(z)=\sum_{s=1}^{s} \pi_{s} U_{s}\left(y+\tilde{y}_{s}-z\right)
$$

is the maximal utility at premium level $z$, and let $K=K(z)$ denote the upper bound in (ii) of Theorem I. Finally, set

$$
K_{v}(z)=\sum_{s=1}^{s} \pi_{s} U_{s}^{\prime}\left(y+\tilde{y}_{s}-z\right)
$$

\section{Theorem 3}

$U^{\prime}(z)$ equals $K(z)-K_{v}(z)$ and is a non-increasing function.

\section{Proof}

Let $z_{1}, z_{2}$ be any two numbers, and let $\tilde{y}_{s}^{(i)}$ denote the optimal coverage for state $s$ if the total premium should be $z_{i}(i=\mathrm{I}, 2)$. Using the concavity from below of $U_{s}$ and property (ii) in Theorem I, we find that

$$
\begin{aligned}
& U_{s}\left(y+\tilde{y}_{s}^{(2)}-z_{2}\right)-U_{s}\left(y+\tilde{y}_{s}^{(1)}-z_{1}\right) \\
\leq & U_{s}^{\prime}\left(y+\tilde{y}_{s}^{(1)}-z_{1}\right) \cdot\left(\tilde{y}_{s}^{(2)}-\tilde{y}_{s}^{(1)}+z_{1}-z_{2}\right) \\
\leq & \frac{q_{s}}{\pi_{s}} K\left(z_{1}\right) \cdot\left(\tilde{y}_{s}^{(2)}-\tilde{y}_{s}^{(1)}\right)-U_{s}^{\prime}\left(y+\tilde{y}_{s}^{(1)}-z_{1}\right) \cdot\left(z_{2}-z_{1}\right) .
\end{aligned}
$$

Multiply both sides by $\pi_{s}$, and summing over $s$, we obtain the inequality

$$
U\left(z_{2}\right)-U\left(z_{1}\right) \leq\left(K\left(z_{1}\right)-K_{v}\left(z_{1}\right)\right) \cdot\left(z_{2}-z_{1}\right)
$$

By interchanging the roles of $z_{1}$ and $z_{2}$, and inverting the sign, a lower bound is obtained for $U\left(z_{2}\right)-U\left(z_{1}\right)$. Finally, assume $z_{1}<z_{2}$.

Then these two inequalities can be written as follows.

$$
K\left(z_{2}\right)-K_{v}\left(z_{2}\right) \leq \frac{U\left(z_{2}\right)-U\left(z_{1}\right)}{z_{2}-z_{1}} \leq K\left(z_{1}\right)-K_{v}\left(z_{1}\right) .
$$

Monotonicity of $K(z)-K_{v}(z)$ is seen immediately from (I9), 
and the rest of theorem 3 follows by taking the limit for $z_{2} \rightarrow z_{1}$. Now observe the following: Let o $\leq \widetilde{z} \leq y$ be the premium spent in the optimal solution $\tilde{y}_{1}, \tilde{y}_{2}, \ldots \tilde{y}_{S}$ of problem B (i.e. $\tilde{z}=\sum_{s=1}^{s} q_{s} \tilde{y}_{s}$ ). For this $\tilde{z}$ problem A must have the same solution as problem B and we conclude, that the two bounds appearing in the characterization of the solutions must be the same, hence

$$
K(\tilde{z})=K_{v}(\tilde{z}) \text {. }
$$

On the other hand theorem 3 leads to the following

\section{Corollary}

$$
\text { If } \quad \begin{array}{lll}
K(0) \leq K_{v}(0) & \text { then } & \tilde{z}=0 \\
K(y) \geq K_{v}(y) & \text { then } & \tilde{z}=y
\end{array}
$$

otherwise let $z$ satisfy

$$
K(z)=K_{v}(z) \quad \text { then } \quad \tilde{z}=z
$$

Based on this corollary and the monotonicity of $K(z)-K_{v}(z)$, $0 \leq z \leq y$ one may find $\breve{z} \neq 0$ by gradually increasing the level $z$ of premium spent until $K(z)-K_{v}(z)=0$, or if this does not happen for $z \leq y$, by putting $\tilde{z}=y$.

Note

It is sometimes more convenient, to follow the above procedure until the quotient $\frac{K(z)}{K_{v}(z)}$ reaches $\mathrm{I}$. To justify this alternative, we also prove that $\frac{K(z)}{K_{v}(z)}$ is nonincreasing $\left(\frac{K_{v}(z)}{K(z)}\right.$ nondecreasing $)$ for $0 \leq z \leq y$.

\section{Proof}

Let $N=N(z)$ denote the set of indices for which $\tilde{y}_{\delta}=0$. Then

$$
K_{v}(z)=K(z)\left(\sum_{\alpha \alpha_{N}}^{\Sigma} q_{s}\right)+\sum_{s \varepsilon_{N}}^{\Sigma} \pi_{s} U_{s}^{\prime}(y-z) \text {, }
$$

and therefore

$$
\frac{K_{v}(z)}{K(z)}=\sum_{\varepsilon f_{N}} q_{s}+\frac{\sum \pi_{s} U_{s}^{\prime}(y-z)}{K(z)} .
$$

Since $\frac{\pi_{s} U_{s}^{\prime}(y-z)}{K(z)} \leq q_{s}$ for $s \in N$, this shows that $K_{v}(z) / K(z)$ is 
a nondecreasing function (the numerator in the last expression is a nondecreasing function, while $K(z)$ is nonincreasing).

In the following the procedure for finding the optimum in problem $B$ is explicitly carried out for

exponential utility (Section 7)

quadratic utility (Section 8).

\section{Exponential Utility}

Let $U_{s}(x)=\mathrm{I}-e^{-\alpha\left(x-y_{*}^{*}\right)}, U_{s}^{\prime}(x)=\alpha e^{\alpha y_{s}^{*}} e^{-\alpha x}$. You may interpret $y_{s}^{*}$ as the "need for money" in state $s$. Suppose then $y$ sufficiently large, such that the following property holds for the optimum $\tilde{y}_{1}, \tilde{y}_{2}, \ldots \tilde{y}_{S}$ of problem B (according to theorem 2).

$$
\frac{\pi_{s}}{q_{s}} e^{\alpha \tilde{y}^{*}} e^{-\alpha \tilde{y}} \leq \sum_{j} \pi_{j} e^{\alpha y_{j}^{*}} e^{-\alpha \tilde{y}_{j}} \quad \begin{aligned}
& \text { for all } s, \text { with strict in- } \\
& \text { equality only allowed if } \\
& \tilde{y}_{s}=0 .
\end{aligned}
$$

With the notation

and

$$
\pi_{s}^{*}=\pi_{s} e^{\alpha y *}
$$

$$
C_{s}\left(y_{1}, y_{2}, \ldots y_{S}\right)=\frac{\frac{\pi_{s}^{*}}{q_{s}} e^{-\alpha y}}{\sum_{j=1}^{s} \pi_{j}^{*} e^{-\alpha y_{j}}}
$$

(22) becomes

$$
C_{s}\left(\tilde{y}_{1}, \tilde{y}_{2}, \ldots \tilde{y}_{s}\right) \leq \mathrm{I} \mid \begin{aligned}
& \text { for all } s, \text { with strict in- } \\
& \text { equality only allowed if } \\
& \tilde{y}_{s}=\mathrm{o} .
\end{aligned}
$$

Abbreviate $\hat{z}$ for $\sum_{j=1}^{s} q_{j} \tilde{y}_{j}$. (25) may hold for $z=0$ and then $\check{z}=0$. Otherwise, increasing gradually the premium level $z$ and adjusting $y_{1}, y_{2}, \ldots y_{s}$ at each level $z$ according to the solution of problem $\mathrm{A}, \max C_{s}$ will monotonically decrease until it reaches $\mathrm{I}$ at $z=\hat{z}$. (See note after theorem 3.) Observe that in the exponential case the ordering

$$
C_{1}\left(y_{1}, y_{2}, \ldots y_{S}\right) \geq C_{2}\left(y_{1}, y_{2}, \ldots y_{S}\right) \geq \ldots \geq C_{S}\left(y_{1}, y_{2} \ldots y_{s}\right)
$$

never changes during this process.

Let then $m$ be the number of states, which are insured in the optimal solution of $\mathrm{B}$ (number of variables $\tilde{y}_{s}$ different from o in $(25)$ ). 
From (2I) we have

$$
K_{v}(\tilde{z})=\sum_{j=1}^{s} \pi_{j}^{*} e^{-x \tilde{y}}=K(\tilde{z}) \sum_{j=1}^{m} q_{j}+\sum_{j=m+1}^{s} \pi_{j}^{*}
$$

and hence from the corollary of theorem 3

$$
\begin{gathered}
\mathrm{I}=\sum_{j=1}^{m} q_{j}+\frac{\mathrm{I}}{K(\bar{z})} \sum_{j=m+1}^{s} \pi_{j}^{*} \\
\frac{\mathrm{I}}{K(\bar{z})}=-\frac{\mathrm{I}-\sum_{j=1}^{m} q_{j}}{\sum_{j=m+1}^{s} \pi_{j}^{*}}
\end{gathered}
$$

therefore (recall $K(\tilde{z})=\frac{\pi_{s}^{*}}{q_{s}} e^{-\alpha \tilde{y}}$ for $\left.s=\mathrm{I}, 2, \ldots m\right)$

$$
\begin{aligned}
\alpha \tilde{y}_{s}= & \log \frac{\pi_{s}^{*}}{q_{s}}+\log \frac{\mathrm{I}}{K(\tilde{z})}= \\
& \log \frac{\pi_{s}^{*}}{q_{s}}+\underbrace{\log \left(\mathrm{I}-\sum_{i=1}^{\sum} q_{j}\right)-\log \underbrace{\sum_{i=m}^{s} \pi_{j}^{*}}_{j=m+1}}_{\Delta m}
\end{aligned}
$$

for $s \leq m$.

The optimal $m$ is found as the first index for which

$\frac{\pi_{m+1}^{*}}{q_{m+1}} \frac{\mathrm{I}-\sum_{j=1}^{m} q_{j}}{\sum_{j=m+1}^{s} \pi_{j}^{*}} \leq \mathrm{I}$ or equivalently $\log \frac{\pi_{m+1}^{*}}{q_{m+1}}+\Delta_{m} \leq 0$

It is easily checked, that this condition also applies if $m=0$.

Numerical Examples (In all examples the exponent $\alpha=\mathrm{I0}^{-2}$ )

First example

$\begin{array}{lccccc}s & I & 2 & 3 & 4 & 5 \\ y_{s}^{*} & \text { I000 } & \text { IOO } & 50 & \text { I0 } & 5 \\ \pi_{s} & 0 . \mathrm{I} & 0.2 & 0.3 & 0.2 & 0.2 \\ q_{s} & 0.3 & 0.3 & 0.3 & 0.3 & 0.3 \\ \pi_{s}^{*} & 2202.65 & .544 & 0.495 & 0.22 \mathrm{I} & 0.2 \mathrm{IO} \\ \frac{\pi_{s}^{*}}{q_{s}} & 7342 . \mathrm{I} 6 & \mathrm{I} .8 \mathrm{I}_{3} & \mathrm{I} .65 & 0.737 & 0.7\end{array}$




$\begin{array}{lrl}\mathrm{I}-\sum_{j=1}^{-1} q_{j} & \text { I } & 0.7 \\ \sum_{j=s}^{s} \pi_{j}^{*} & 2204 . \mathrm{I} 2 & \mathrm{I} .470 \\ \operatorname{check}(27) & 3.33 & 0.863\end{array}$

Hence only state $I$ is insured and from (26) $\tilde{y}_{1}=8 \mathrm{I} 5.95$

$$
q_{1} \tilde{y}_{1}=244.78 \text {. }
$$

\section{Second example}

Insurance becomes "horribly expensive" for $s=\mathrm{I}$, otherwise same as in first example.

\begin{tabular}{|c|c|c|c|c|c|}
\hline$s$ & $I$ & 2 & 3 & 4 & 5 \\
\hline$y_{s}^{*}$ & IOOO & IOO & $5^{\circ}$ & IO & 5 \\
\hline$\pi_{s}$ & O.I & 0.2 & 0.3 & 0.2 & 0.2 \\
\hline$q_{s}$ & $I$ & 0.3 & 0.3 & 0.3 & 0.3 \\
\hline$\pi_{s}^{*}$ & 2202.65 & 0.544 & 0.495 & $0.22 \mathrm{I}$ & $0.2 \mathrm{IO}$ \\
\hline$\frac{\pi_{s}}{q_{s}}$ & 2202.65 & I.8I3 & I. 65 & 0.737 & 0.7 \\
\hline $\begin{array}{l}\sum_{j=-} \pi_{\mathrm{j}}^{*} \\
\text { check }(27)\end{array}$ & $\begin{array}{l}2204.12 \\
<I\end{array}$ & & & & \\
\hline
\end{tabular}

Hence now no insurance is bought at all!

\section{Third example}

The "insurance need" is eliminated in state $s=\mathrm{I}$, otherwise still the same as before.

$\begin{array}{lcclll}s & 2 & 3 & 4 & 5 & I \\ y_{s}^{*} & \text { I00 } & 50 & \text { Io } & 5 & 0 \\ \pi_{s} & 0.2 & 0.3 & 0.2 & 0.2 & 0 . \mathrm{I} \\ q_{s}^{*} & 0.3 & 0.3 & 0.3 & 0.3 & 0.3 \\ \pi_{s}^{*} & 0.544 & 0.495 & 0.22 \mathrm{I} & 0.2 \mathrm{IO} & 0 . \mathrm{I} \\ \pi_{s}^{*} & \text { I.8I3 } & \text { I.65 } & 0.737 & 0.7 & 0.333 \\ q_{s} & \text { I.I55 } & \text { I.I26 } & 0.555 & & \end{array}$

Hence insurance on $s=2$ and $3 \quad \tilde{y}_{2}=3$ I.I $7 q_{2} \tilde{y}_{2}=9.35$ 


\section{Quadratic Utility}

In this section

$$
\begin{aligned}
& U_{s}(x)=\alpha\left(x-y_{s}^{* *}\right)-\frac{\left(x-y_{s}^{* *}\right)^{2}}{2} ; x-y_{s}^{* *} \leq \alpha \\
& U_{s}^{\prime}(x)=\alpha+y_{s}^{* *}-x
\end{aligned}
$$

The condition corresponding to $(22)$ in Section 7 is then

$\frac{\pi_{s}}{q_{s}}\left(\alpha+y_{s}^{* *}-y-\tilde{y}_{s}+\sum_{j} q_{j} \tilde{y}_{j}\right) \leq \alpha+\bar{y}_{\delta}^{* *}-y-\overline{\widetilde{y}}+\sum_{j} q_{j} \tilde{y}_{j}(28)$ for all $s$, with strict inequality only allowed if $\tilde{y}_{s}=0$

Abbreviations

$$
\begin{aligned}
\bar{y}^{* *} & =\sum_{j} \pi_{j} y_{j}^{* *} \\
\bar{y} & =\sum_{i} \pi_{j} \tilde{y}_{j}
\end{aligned}
$$

Redefine

$$
\begin{gathered}
\alpha+y_{s}^{* *}-y=y_{s}^{*} \quad \text { and you obtain } \\
\frac{\frac{\pi_{s}}{q_{s}}\left(y_{s}^{*}-\tilde{y}_{s}+\sum_{j} q_{j} \tilde{y}_{j}\right)}{\bar{y}^{*}-\overline{\bar{y}}+\sum_{j} q_{j} \tilde{y}_{j}} \leq \mathbf{I}
\end{gathered}
$$

for all $s$, with strict inequality only allowed if $\tilde{y}_{s}=0$

Observe that as long as the numerator of the left side in (29) is positive, we are in the region where $U_{s}^{\prime}$ is positive. The numbering of the sides is defined in decreasing order of

$$
C_{s}=\frac{\pi_{s}}{q_{s}} \frac{y_{s}^{*}}{\bar{y}_{s}} \text {, hence } C_{1} \geqslant C_{2} \geqslant \ldots \geqslant C_{S}
$$

These quantities are the inital values at $y_{1}=y_{2}=\ldots=y_{S}=\mathrm{o}$ of the functions

$$
C_{s}\left(y_{1}, y_{2}, \ldots y_{S}\right)=\frac{\frac{\pi_{s}}{q_{s}}\left(y_{s}^{*}-y_{s}+\Sigma q_{j} y_{j}\right)}{y^{*}-\bar{y}+\Sigma q_{j} y_{j}}
$$

We again gradually increase $z=\sum_{j} q_{j} y_{j}$ and for each $z$ adapt $y_{1}, y_{2}, \ldots y_{S}$ according to the solution of problem $\mathrm{A} ; \max C_{s}$ will then again monotonically decrease to $I$, but unfortunately the ordering of the $C_{s}\left(y_{1}, y_{2}, \ldots y_{s}\right)$ (for those $s$ which are not yet 
insured) may change! So while it is clear that insurance, if any, must always be bought on $s=\mathrm{I}$, we must if necessary try several combinations of other states to find out the optimum.

Numerical Examples

First example

\begin{tabular}{|c|c|c|c|c|c|c|}
\hline$s$ & $I$ & 2 & 3 & 4 & 5 & \\
\hline$s$ & IOOO & IOO & $5^{0}$ & IO & 5 & \\
\hline$\tau_{s}$ & O.I & 0.2 & 0.3 & 0.2 & 0.2 & $=\mathrm{I} 38$ \\
\hline$q_{\delta}$ & 0.3 & 0.3 & 0.3 & 0.3 & 0.3 & \\
\hline$C_{s}$ & $2.4 \mathrm{I} 5$ & 0.483 & 0.362 & 0.048 & 0.024 & \\
\hline
\end{tabular}

We try to insure state number I only. If this does achieve an optimum we must have

$$
C_{1}\left(y_{1}, 0,0, \ldots 0\right)=\frac{\mathrm{I}}{3} \frac{\mathrm{I000}-y_{1}+0.3 y_{1}}{\mathrm{I} 38-0 . \mathrm{I} y_{1}+0.3 y_{1}}=\mathrm{I}
$$

from which we find

$$
\begin{aligned}
y_{1} & =450.77 \\
q_{1} y_{1} & =I 35.23
\end{aligned}
$$

It remains to be checked whether $C_{s}\left(y_{1}, 0,0, \ldots o\right) \leq \mathrm{I}$ for $s \geq 2$

$$
\begin{gathered}
C_{2}\left(y_{1}, 0,0, \ldots o\right)=\frac{2}{3} \frac{100+\mathrm{I} 35.23}{228 . \mathrm{I} 5}=0.69 \\
\left.C_{3}\left(y_{1}, 0,0, \ldots o\right)=\frac{3}{3} \frac{50+\mathrm{I} 35.23}{228 . \mathrm{I} 5}=0.8 \mathrm{I} \text { (has surpassed } C_{2} !\right)
\end{gathered}
$$

As states 4 and 5 have the same probabilities and premiums as state 2 their $C$-values must be lower than that of state 2 also. This shows that just insuring state $\mathbf{I}$ with the above amounts is optimal.

\section{Second example}

If we change in the first example only $q_{1}$ from 0.3 to I (insurance on the state insured in the first example becomes "horribly expensive"), then all initial $C$-values drop below I which means that no insurance should be bought. 


\section{Third example}

"Insurance need" in state $I$ is eliminated (i.e. $y_{1}^{*}=0$ ). Otherwise same as first example.

\begin{tabular}{lccclll}
$s$ & 2 & 3 & 4 & 5 & \multicolumn{1}{c}{} & \\
$y_{s}^{*}$ & I00 & 50 & Io & 5 & 0 & \\
$\pi_{s}$ & 0.2 & 0.3 & 0.2 & 0.2 & $0 . \mathrm{I}$ & $\bar{y}^{*}=38$ \\
$q_{s}$ & 0.3 & 0.3 & 0.3 & 0.3 & 0.3 & \\
$C_{s}$ & $\mathrm{I} .75$ & $\mathrm{I} .32$ & $0 . \mathrm{I} 8$ & 0.09 & 0 &
\end{tabular}

It is obvious that some insurance must be bought, certainly on $s=2$ and probably also on some other states, $s=3$ being a very likely candidate.

We try to find an optimal solution, where $y_{2}$ and $y_{3}$ are different from zero

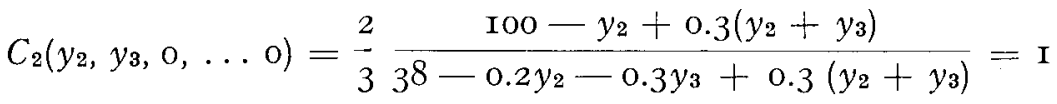

$$
\begin{aligned}
& \text { or } 860-14 y_{2}+3 y_{3}=0 \\
& C_{3}\left(y_{2}, y_{3}, 0, \ldots o\right)=\frac{50-y_{3}+0.3\left(y_{2}+y_{3}\right)}{38-0.2 y_{2}-0.3 y_{3}+0.3\left(y_{2}+y_{3}\right)}=\mathrm{I} \\
& \text { or } I 20-7 y_{3}+2 y_{2}=0 \\
& y_{2}=69.35 \quad y_{3}=36.96 \\
& q_{2} y_{2}=20.80 \quad q_{3} y_{3}=I I .09 \quad \text { total premium } \quad 3 I .89
\end{aligned}
$$

We must check that $C_{4}\left(y_{2}, y_{3}, 0,0,0\right) \leq \mathrm{I}$. This check suffices since $\frac{\pi_{s}}{q_{s}} \leq \frac{\pi_{4}}{q_{4}}$ for $s=5, \mathrm{I}\left(C_{5}\right.$ and $C_{1}$ will then automatically be below $\left.\mathrm{I}\right)$.

Check: $C_{4}\left(y_{2}, y_{3}, 0,0,0\right)=\frac{2}{3} \frac{\text { Io }+31.89}{38-24.96+31.89}=0.62$,

which proves optimality.

\section{Literature}

[I] Arrow, K. J., (1964), The Role of Securities in the Optimal Allocation of Risk Bearing. Review of Economic Studies (translation of original paper in French, 1953).

[2] Arrow, J. K., (1974), Optimal Insurance and Generalized Deductibles Scandinavian Actuarial Journal.

[3] Borch, K., (1978), Problems in the Economic Theory of Insurance. The ASTIN Bulletin, Io, I-II. 